\title{
Gradhiva
}

GRADHIV

Revue d'anthropologie et d'histoire des arts

$10 \mid 2009$

Présence africaine

\section{Alioune Diop, l'un des pères de la civilité démocratique mondiale}

Témoignage de l'écrivain franco-haïtien René Depestre

\section{(2) OpenEdition}

Journals

Édition électronique

URL : http://journals.openedition.org/gradhiva/1574

DOI : 10.4000/gradhiva. 1574

ISSN : 1760-849X

Éditeur

Musée du quai Branly Jacques Chirac

Édition imprimée

Date de publication : 4 novembre 2009

Pagination : 164-169

ISBN : 978-2-35744-012-8

ISSN : 0764-8928

Référence électronique

"Alioune Diop, l'un des pères de la civilité démocratique mondiale », Gradhiva [En ligne], 10 | 2009, mis en ligne le 03 février 2010, consulté le 20 avril 2019. URL : http://journals.openedition.org/ gradhiva/1574 ; DOl : 10.4000/gradhiva.1574

(c) musée du quai Branly 


\title{
Alioune Diop, l'un des pères de la civilité démocratique mondiale
}

\author{
Témoignage de l'écrivain franco-haïtien René Depestre
}

J'ai dû entendre parler d'Alioune Diop, la première fois, à Paris, chez Aimé Césaire (au 10 de la rue de l'Odéon) lors des rencontres du dimanche après-midi en compagnie de René Menil, Suzanne Roussi, Michel Leiris, André Lhote, Jean Cassou, Wifredo Lam...

À la même époque, Léon-Gontran Damas et Frantz Fanon m’ont aussi parlé d'Alioune Diop et de son projet de revue, qui devait prendre corps à la fin de 1947.

Je reçus ensuite une lettre d'Alioune Diop (au début 1948) par laquelle il m'invitait à rejoindre la rédaction de la revue, en benjamin de l'équipe. Conscient de la ténuité de mes connaissances d'étudiant (à Sciences Po et à la Sorbonne), auteur de deux plaquettes de poèmes, je déclinai la flatteuse proposition.

Vint alors ma rencontre avec Alioune Diop, rue des Écoles. Il me fit un accueil d'emblée fraternel : je fus aussitôt frappé par sa courtoisie, sa maîtrise de soi, la sobriété de sa force intérieure, sa rigueur intellectuelle et son haut coefficient de civilité. Autant de qualités chez un homme qui fascinèrent le garçon encore tout feu tout flamme que j'étais. Je lui promis d'être un lecteur assidu de Présence Africaine, et plus tard, après les études universitaires, d'apporter ma modeste collaboration à son œuvre décoloniale.

Mais, de 1948 à 1955, sept ans durant, surtout à partir de fin 1950, je fus pris dans un tourbillon très caractéristique de la guerre froide qu'on vivait alors.

Fin 1950 et 1951 : je vis un exil incertain, à Prague, sous le coup d'une mesure d'expulsion de la France. À cette époque, Césaire préface ma plaquette Végétations de clarté (Seghers).

1952 : passage en Italie, retour en France, nouvelle expulsion de France.

1952-1953 : départ d'Italie pour le Chili (via le Brésil et l'Argentine), séjour au Chili et en Argentine.

1953-1955 : séjour au Brésil (Rio et São Paulo).

Pendant ces temps aventureux, vivant au Brésil sous le faux nom de Walter Miranda (commis-voyageur en librairie), je n'ai pas arrêté de lire Présence Africaine et de réfléchir à l'avenir de la négritude, fidèle en cela à Césaire, Damas, Fanon, Henri Bangou et autres fortes relations des années parisiennes (1946-1950). De même, je gardai les contacts avec mes amis du Parti communiste français, notamment l'équipe de l'hebdomadaire Les Lettres françaises, dirigé par Aragon.

En 1955, Aragon ouvre un grand débat sur la poésie nationale. Poète de langue française, citoyen d'une «nation » d'expression française, l'argumentaire d'Aragon m'intéresse. Dans une lettre datée de São Paulo, je fais part au poète Charles Dobzynski de mon intérêt pour une discussion à laquelle participaient de nombreux poètes français (Tzara, Guillevic, Claude Roy, Marcenac, Dobzynski, Pierre Seghers, etc.). Dobzynski était rédacteur aux Lettres françaises. À ce titre, il communique ma lettre à Aragon, qui la publie entièrement en première page de son hebdo. 
Aimé Césaire prend très mal mon texte. Il réagit vivement avec un très beau poème intitulé À René Depestre, poète haïtien (éléments d'un art poétique). Le poème a été repris sous le titre Le verbe marroner (Euvres complètes, 1976). Le poème de Césaire soulève un grand enthousiasme chez les étudiants qui entourent Présence Africaine. La revue s'empare opportunément du "scandale» que constitue ma lettre. Le poète révolutionnaire que Césaire lui-même avait préfacé en 1951 (quatre ans auparavant, chez Seghers) se rangeait sous la bannière réactionnaire, ringarde, du sonnet et des autres formes obsolètes de la prosodie française, sous la houlette de Louis Aragon. Un soir, un débat est organisé au siège de Présence Africaine rue des Écoles. Césaire y prend la parole pour condamner, cette fois dans une prose cinglante, ce qu'il appelle mes « positions assimilationnistes ». Il stigmatise carrément ma participation à une opération qui avait un relent colonialiste... En clair, il me surprenait en flagrant délit de complicité avec une campagne du vers français qui, au fond, relevait à ses yeux de la rhétorique insolente et dérisoire d'un maître attelé, de toute sa superbe hexagonale, à une typique histoire de "grand blanc» de l'alexandrin et du sonnet !

"Que le poème tourne bien ou mal sur l'huile de ses gonds fous-t'en Depestre, laisse dire Aragon " (vers qui ont disparu dans les éditions complètes, le titre initial également).

Été 1955 : retour à Paris. Je découvre, en même temps, le fameux poème de Césaire, le texte en prose, climat polémique, une discussion extraordinaire à laquelle participe toute la jeune intelligentsia réunie autour d'Alioune Diop, au siège de Présence Africaine.

En réalité, Aimé Césaire avait saisi le débat suscité par la campagne d'Aragon pour régler des comptes, non seulement avec l'auteur du Paysan de Paris, mais avec le PCF. C'était l'aboutissement d'un contentieux politique et idéologique de fond. J'étais au courant des difficultés de Césaire, député communiste de la Martinique, avec l'appareil stalinien des Thorez et Duclos. D'ailleurs, moins d'un an plus tard, Césaire devait rompre avec le PCF par sa célèbre Lettre à Maurice Thorez (24 octobre 1956). Par ma personne interposée, Césaire, dès l'été 1955 , manifesta sa volonté de rupture avec les thèses stalinistes du parti, pas seulement en matière de prosodie traditionnelle, mais avec l'impérialisme culturel par lequel le PCF manifeste sa mentalité assimilationniste et sa foi dans «la supériorité omnilatérale de l'Occident » et dans la Civilisation avec un grand C. Césaire rompait, une fois pour toutes, avec la sinistre école primaire de Staline qu'était l'ensemble du mouvement communiste international. Césaire réprouvait fermement l'usage terroriste que les partis communistes du monde entier faisaient du marxisme et du communisme. "Que la doctrine et le mouvement soient faits pour les hommes, non les hommes pour la doctrine et pour le mouvement. » Césaire mettait brutalement fin au magistère politique que le PCF exerçait à l'égard des peuples colonisés. Césaire abandonnait les vieilles routes communistes qui avaient conduit le mouvement ouvrier à l'imposture, à l'ignominie, à la tyrannie, au crime. C'était là le fond du débat, apparemment esthétique, littéraire, autour de l'épître, du sonnet et de l'alexandrin.

Mais durant l'été 1955, un an avant l'adieu de Césaire au stalinisme et au goulag, dans la revue Présence Africaine, on devait plutôt débattre des rapports de la négritude et de la question nationale aux Caraïbes et dans les Afriques. Pour mon humble part, pris à parti par un grand poète qui avait toute ma confiance et mon admiration, j'essayai tant bien que mal de m'en sortir par quarante pages de réponse à Aimé Césaire publiées par Présence Africaine.

Aujourd'hui, un demi-siècle après, ce texte me paraît passablement d'un marxisme laborieux et peu convaincant... Le mérite du débat de 1955, apparemment autour des idées académiques d'Aragon, c'est que, grâce à la riposte de Césaire, il nous offrit l'occasion d'un rapprochement sans précédent.

Dès lors, je confiai le manuscrit de mon livre Minerai noir à Présence Africaine. Je découvris l'équipe qui entourait le précieux travail d'Alioune Diop: Jacques Howlett, Thomas Diop, Iwiyé Kala-Lobé, Paulin Joachim, Christiane Yandé Diop, la dynamique compagne d'Alioune, David Diop, Mario de Andrade, Jacques Rabemananjara. 
Cette équipe était en condition de préparer le premier « Congrès des écrivains noirs » qui occupait depuis longtemps l'esprit toujours en ébullition d'Alioune Diop. Le débat de 1955 devait donc marquer un nouveau départ et une nouvelle cohésion autour de la revue qui rendit possible la réussite du congrès de la Sorbonne en septembre 1956.

Lors de la préparation du congrès, j'eus des séances de travail avec Alioune Diop, afin de le recycler dans les réalités culturelles d'Haïti. Il comptait sur une importante délégation haïtienne : ce fut le cas. Présidée par Jean Price-Mars (qui devait être élu président de nos travaux de la Sorbonne), on comptait dans ses rangs Jacques Stephen Alexis, Albert Mangones, Emmanuel C. Paul, Émile Saint Lot, René Piquion, Gérard Bissainthe et moi. On m’a posé souvent la question : pourquoi n'a-t-on pas entendu votre voix durant les débats de septembre 1956 à la Sorbonne, vous qui, l'année précédente, aviez été si prolixe lors du débat sur la poésie nationale? C'est l'occasion pour moi d'en parler pour la première fois dans ce témoignage sur Alioune Diop. Le célèbre rapport de Nikita Sergeïevitch Khrouchtchev avait précédé le Premier Congrès des écrivains noirs. En 1956, la parole du dirigeant soviétique arriva comme une épreuve sismique dans mes jours, d'autant que j'avais pu voir en 1951 la partie émergente de l'iceberg stalinien. Durant mon séjour à Prague, j'avais adressé une lettre de quarante pages à Aimé Césaire pour lui dire ma consternation devant le terrorisme d'État, que la propagande de notre mouvement communiste présentait comme "un modèle de démocratie populaire (sic) ».

Ce qui me retint en 1951 de rompre avec le communisme, ce fut ma rencontre avec Pablo Neruda et Jorge Amado, qui m'invitèrent à les rejoindre au Chili et au Brésil. «Nous ferons autrement la révolution ", me dirent-ils. Le rapport Khrouchtchev confirmait déjà qu'on était en présence d'un naufrage sans rémission de la révolution d'Octobre. Alors je confiai à Alioune Diop que je pensais saisir la tribune qui m'était offerte au Congrès des écrivains noirs de la Sorbonne pour rompre avec éclat avec le PCF et le stalinisme. Je mis sous ses yeux le texte de rupture que j'avais déjà préparé, et qui était une sorte de lettre ouverte à Nikita Khrouchtchev. Mon texte était un brûlot de jeune homme en colère. Il impressionne Alioune par sa violence verbale, d'un lyrisme incandescent. C'était la parole d'un poète nègre déçu et blessé à mort. Je faisais entrer la guerre froide dans l'amphithéâtre Descartes de nos discussions.

Alioune Diop, toujours maître de soi, parut toutefois contrarié... Il voyait par terre le consensus qui lui permettait de réunir, sous le même toit universitaire, les antagonismes qui, dans des conditions manichéennes, divisaient les intelligentsias du monde entier. Après la lecture de mon texte, il prit sa tête entre ses mains : "Tu vas mettre le feu aux poudres, me dit-il. Tu vas politiser le débat. La délégation des États-Unis te fera une fête. Notre inventaire des valeurs culturelles noires ne pourra plus compter sur la sérénité et la tolérance de nos travaux. Je te laisse cette nuit pour réfléchir à tout cela. »

Rentré à la maison, et après réflexion, je pensai que le consensus obtenu alors par Alioune Diop, après des années de contact avec les intellectuels de tous les horizons et dans la conjoncture où se trouvait notre combat décolonial, était notre lien le plus précieux. En aucun cas, on ne devait le mettre en péril. Alors, à la place du missile destiné au camarade NK..., j'écrivis dans un seul souffle un long poème intitulé La Petite Lampe sur la mer. Je le dédie à mes frères noirs réunis à la Sorbonne...

Le lendemain, je vis des larmes monter aux yeux d'Alioune Diop à la lecture du poème qui remplaçait la fusée intellectuelle de l'amour déçu. La Petite Lampe sur la mer paraîtra, dis-je, dans Les Lettres françaises d'Aragon. Voilà le grand secret que je devais partager avec Alioune Diop. J'avais alors bien fait de m'effacer en quelque sorte devant mon aîné Aimé Césaire, qui, par sa lettre du 24 octobre 1956 à Maurice Thorez, devait mille fois mieux que moi proférer orbi et urbi la parole décisive d'adieu de tous les Noirs aux utopies historiques du communisme.

Après cette expérience de ma collaboration avec Alioune Diop, je devais donc publier aux éditions Présence Africaine, en 1957, Minerai noir, et, en 1967, Un arc-en-ciel pour l'Occident chrétien (peut-être mon meilleur livre de poèmes). 


\section{Une intelligentsia noire constituée autour d'Alioune Diop}

Dès les travaux du Premier Congrès des artistes et écrivains noirs réuni à la Sorbonne, du 19 au 22 septembre 1956, à écouter la parole inaugurale d'Alioune Diop, à le voir au travail pour unir dans un projet décolonial consensuel des intellectuels noirs pris séparément dans les antagonismes manichéens de la guerre froide, on pouvait se faire une idée précise de l'originalité de son magistère culturel. À mes yeux, Alioune Diop était l'homme, le grand homme sénégalais, d'une triple vision synoptique et intégrante des réalités du monde noir au xx siècle.

a) Alioune Diop avait une vision synoptique et intégrante de l'aventure historique des peuples noirs après l'esclavage et la colonisation qui avaient décimé, avili, déguisé leur destin dans un carnaval oppressif sans précédent, en leur fabriquant une condition nègre et toute une situation coloniale de Noirs...

b) Alioune Diop avait une vision synoptique et intégrante des nouvelles formes de civilisation que, dans les conditions effroyables de la traite et dans diverses sociétés de plantations des Amériques, leurs descendants surent inventer dans les différentes aires culturelles, les divers empires coloniaux. Les Africains déportés, victimes de la rupture opérée entre les ethnies et les cultures, surent, grâce au marronage et à la créolisation, créer les conditions (toujours précaires) d'une nouvelle identité historique.

c) Alioune Diop avait une vision synoptique et intégrante des actions d'ensemble qu'il fallait mener pour aider les intelligentsias noires, intégrées à diverses cultures nationales de l'Occident impérial après deux siècles d'expériences collectives, à rejoindre la condition humaine universelle que la violence esclavagiste et coloniale leur avait dérobée.

À cette fin grandiose, Alioune Diop avait consacré une exceptionnelle énergie, une intelligence de premier ordre, une capacité d'écoute, de dialogue, de civilité, de tolérance, de tendresse hors du commun, dans une époque où les idéologies dominantes tournaient les individus et les peuples les uns contre les autres. Seul, avec une équipe modeste de collaborateurs, avec des moyens quasi artisanaux, il mit sur pied, tour à tour, une revue, une maison d'édition, une Société africaine de culture. Autour d'un programme de décolonisation de toute une famille de sociétés et de cultures, il sut organiser de multiples colloques et des séminaires, dans tous les domaines de la connaissance moderne, pour éclairer la marche des peuples et des intelligentsias.

Déjà, à la fondation de la revue, c'était une gageure de réunir à Paris un comité de soutien à son projet, des hommes aussi éminents que Gide, Sartre, Camus, Théodore Monod, Claude Julien, le révérend père Maydieu, Michel Leiris, etc., et cela dans un haut lieu de l'Occident chrétien qui était en mesure (et ce fut le cas) de surdéterminer le rayonnement intellectuel de son action.

Aujourd'hui, avec de nombreuses années de recul, on peut dire que l'énergie créatrice d'Alioune Diop créa en France et en Afrique les conditions d'une sorte de renaissance de ce que Sartre devait appeler l'Orphée noir. Avant l'entrée en scène d'Alioune Diop dans les années 1920, soufflait aux États-Unis un semblable esprit de renouveau des esprits, grâce à toute une équipe d'intellectuels nègres, W. E. B. Du Bois, Alain Locke, Langston Hughes, Countee Cullen, sur un fond culturel collectif de jazz, de blues, de negro spirituals. La pensée d'Alioune Diop se situait dans le droit fil d'un mouvement de renaissance qui avait marqué profondément la génération de Senghor, Damas, Césaire. Comme en Amérique, elle avait conduit à la maturité les Jean Price-Mars, Jacques Roumain, Magloire Saint Aude, Jean F. Brierre en Haïti, un C. L. R. James à Trinidad et Tobago, et un Nicolas Guillen et un Regino Pedroso à Cuba.

En 1955, au moment de passer à la préparation des deux congrès décisifs de Paris et de Rome, Alioune Diop pouvait compter sur une brillante intelligentsia présidée par des intellectuels de premier plan comme Richard Wright, Bernard Dadié, Césaire, Senghor, Damas, Price-Mars, Fanon, J. S. Alexis, Georges Lamming, James Baldwin et les talents des nouvelles générations Édouard Glissant, Cheikh Anta Diop, Mongo Beti, David Diop, Joseph Ki- 
Zerbo, Amadou Mahtar, M’Bow, Tchicaya U Tam'si, Henri Lopez et Édouard Mauninck, qui trouvaient les moyens de se faire éditer.

Avec les deux congrès de Paris et de Rome et les deux festivals de Dakar et Lagos, Alioune Diop avait su assurer une liaison féconde de création entre les multiples foyers nègres de la planète, et cela sans être jamais tenté de constituer un mouvement identitaire du type messianique et intégriste.

Alioune Diop, peut-être, dans de meilleures conditions que Senghor ou Césaire, aurait pu, n'était sa rigueur de pensée, céder à la tentation d'un pannégrisme revanchard et haineux doté des têtes chercheuses de la barbarie et du ressentiment. À ses yeux, l'idée de négritude qu'il partageait avec les fondateurs de ce concept anthropologique n'avait rien d'une « essence noire » antérieure aux expériences de l'esclavage et de la colonisation. Diop, nourri dans le meilleur terreau des traditions africaines de son pays wolof, avait su les métisser avec un savoir universitaire, de grand aloi, à la française, c'est-à-dire une pensée décoloniale gonflée d'oxygène et de toutes les valeurs nutritives de la civilité démocratique.

S'il avait hérité intelligemment de l'esprit de renaissance propre au jazz et à la grande pensée lyrique des Noirs américains, il avait également intégré à son savoir libérateur les expériences du panafricanisme, mouvement politique né à Trinidad et Tobago avec Sylvester Williams, fécondé aux États-Unis de Du Bois et que George Padmore devait révéler à K. Nkrumah et aux divers courants décolonisateurs propres aux anciennes colonies britanniques. Diop est l'homme pionnier de la synergie décoloniale entre le Commonwealth britannique et le monde francophone, appelés à jouer un rôle commun dans la Weltanschauung aujourd'hui promise à servir de garde-fou aux graves crises financières et économiques de la mondialisation. Alioune Diop apparaît donc désormais comme l'homme dont le monde va avoir besoin grâce à son haut coefficient d'interdépendance et d'interpénétration des imaginaires qui quadrillent la planète.

Parce qu'il avait une vision synoptique et intégrante des réalités spécifiques du monde noir (issu de l'esclavage et de la colonisation), sa pensée nous offre un modèle méthodologique, pour le nouvel équilibre civilisationnel entre la nature et l'histoire, qui doit aider l'espèce humaine (la famille des humanités) à sortir ensemble de l'actuel morcellement identitaire vers tout un art de créer ensemble des formes laïques de sacralisation des institutions démocratiques.

Alioune Diop, dans la sphère culturelle où il a fonctionné avec succès, montre qu'au lieu de toujours s'opposer il est possible de s'appuyer sur des acquis pour que les cultures deviennent complémentaires les unes des autres.

Alioune Diop m’a paru toujours porteur avec sérénité des valeurs spirituelles, des qualités et de l'énergie créatrice d'un grand homme. Sa vie aura été une aventure étincelante de la culture humaine. Alioune Diop aurait été heureux de voir le sénateur de l'Illinois, Barack Obama, accéder à la première magistrature des États-Unis d'Amérique. «Nous ne pouvons rejoindre les autres, disait-il, au plan des aspirations universalistes qu'après avoir été souverainement nous-mêmes. " Alioune Diop, dans cette voie de réunification de la conscience des humanités, devait faire avancer considérablement les choses, et il faut retenir la haute qualité de son magistère et de sa générosité sans bornes, à l'heure d'évaluer, dans le panorama culturel, les forces des multiples foyers historiques nègres qui ont préparé les conditions de l'avènement d'un leader politique noir à la présidence des États-Unis.

Le leadership de Barack Obama, à l'horizon mondial, ne serait pas riche de tant de promesses de démocratie si, en plus des héritages culturels propres à l'histoire des Noirs des États-Unis, le souci de l'humanisation de la vie en société n'avait pas poussé en avant sur la scène des civilisations des êtres humains de lumière comme le Sénégalais Alioune Diop, capables de faire passer la justice et la fraternité du droit formel des États-Unis à l'universalité concrète d'une civilité démocratique mondiale. 


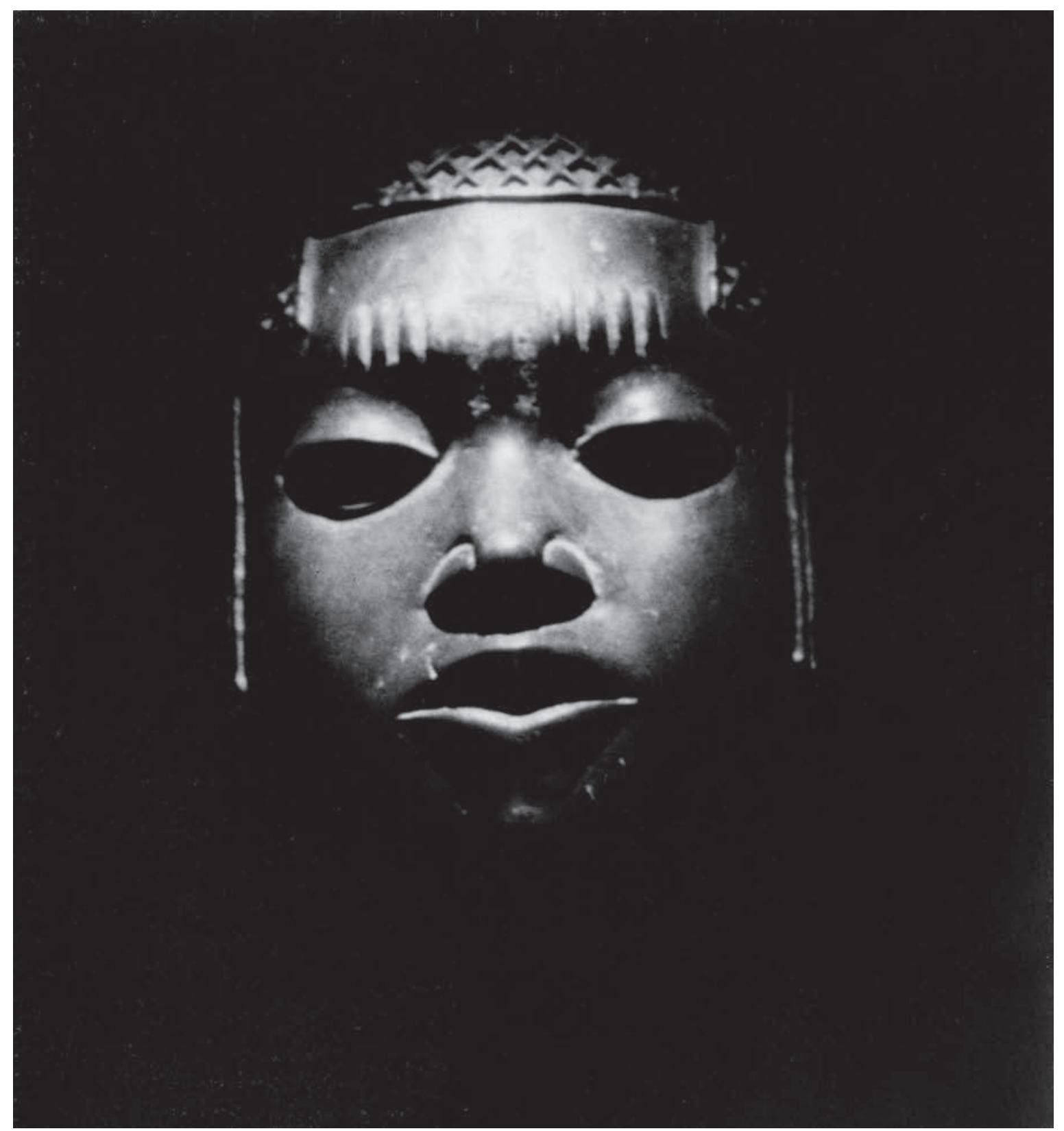

Photogramme du film Les statues meurent aussi de Chris Marker et Alain Resnais, 1953. Iconothèque de la Cinémathèque française ๔) Présence Africaine. 